\title{
Tangence
}

\section{Entre réel et mythe : la souffrance, le désespoir et la tentation du suicide dans le Journal de Charles Juliet}

\section{Michel Braud}

Numéro 45, octobre 1994

Authenticité et littérature personnelle

URI : https://id.erudit.org/iderudit/025827ar

DOI : https://doi.org/10.7202/025827ar

Aller au sommaire du numéro

Éditeur(s)

Tangence

ISSN

0226-9554 (imprimé)

1710-0305 (numérique)

Découvrir la revue

Citer cet article

Braud, M. (1994). Entre réel et mythe : la souffrance, le désespoir et la tentation du suicide dans le Journal de Charles Juliet. Tangence, (45), 83-90.

https://doi.org/10.7202/025827ar d'utilisation que vous pouvez consulter en ligne. 


\section{Entre réel et mythe : la souffrance, le désespoir et la tentation du suicide dans le Journal de Charles Juliet \\ Michel Braud}

I

La littérature personnelle ou autobiographique se veut l'image d'une existence ou de moments d'existence vécus, et s'oppose communément à la fiction qui n'entretient pas comme elle ce rapport au réel. Elle renvoie à un univers physique qui est ou a été, et à des événements qui se sont produits. Elle se définit par le pacte qu'elle met en place lors de la lecture, et qui assure sa lisibilité. Ce pacte atteste de l'existence du réel évoqué, ainsi que du rapport de vérité établi entre ce réel et la représentation présentée dans le texte.

La construction de la représentation du réel se présente certainement le plus simplement dans les textes de mémoires ou de journaux de voyage, parce que ce réel est extérieur au sujet: l'objet du discours a pu être observé par d'autres, et si l'image qu'en donne le mémorialiste n'est pas nécessairement celle qu'aurait pu en avoir le lecteur, elle n'en reste pas moins vraie dans la mesure où l'auteur assure par le pacte avoir bien eu l'intention de donner sa perception du réel observé. Dans le cas de la littérature autobiographique, en revanche, l'objet du discours n'est en général que secondairement extérieur au sujet, la partie la plus importante étant constituée par des évocations de sensations, sentiments ou rêves. Le réel est alors, pour l'essentiel, le moi, dans ce que celui-ci a de fuyant, d'incertain. Et non seulement le plus souvent le réel se confond avec le moi, mais de plus il se confond avec la perception qu'en a le sujet; le désir ou le désespoir ne semblent pas dissociables, pour le diariste, de la perception qu'il en a.

Pourtant, pour celui-ci, le sentiment est un événement qui a lieu, dont la réalité ne peut pas être contestée, voire dont la réalité s'impose à la conscience. Ainsi, chez Charles Juliet, la peur, l'ennui, l'angoisse ou la souffrance ne peuvent qu'être constatés : 
84

Quand la peur déferle, tu fuis, vas traîner dans les rues. Tu n'as pas assez de force pour oser affronter un tel état. ${ }^{1}$

Ou un peu plus tard:

L'ennui. Seconde après seconde, lente, lourde, un peu plus loin dans l'atroce, tu erres en toi, te saccages. Sans fin. Dans un dégoût accru. ${ }^{2}$

La peur est un objet de perception qui semble exister indépendamment du moi, et à distance de lui. Et l'atroce n'est qu'un lieu, l'espace arpenté par la conscience-regard. Ces procédés de mise à distance élèvent la perception et le sentiment au statut d'objet de la conscience, et distinct d'elle, ou même en font le seul objet qui puisse être appréhendé à ce moment-là; la peur et l'atroce, comme ailleurs la souffrance, l'ennui ou l'angoisse, sont à la fois expérience, donc événement qui a lieu, et, par la mise à distance, sont à l'image du monde extérieur perçu par la conscience, s'imposent comme réels par le regard que le moi porte sur eux.

Le diariste accentue cette impression en présentant sa souffrance comme une instance dont il serait l'objet, et qui l'enferme dans une immobilité mortifère.

La souffrance progresse, ronge, ronge, me conduit à l'épuisement. Et alors je ne souffre plus, ne pense plus, ne puis plus me mouvoir, ne suis plus que pierre. Définitivement inerte, aride, coupé de toute vie, toute possibilité de naissance. Face à face avec ma mort. ${ }^{3}$

Ou encore la distance qui s'instaure entre son existence et lui-même fait de son ennui, son angoisse ou son désespoir un spectacle auquel il assiste et dont il n'arrive pas à rendre compte:

Ce que j'écris semble ne pas me refléter, se situer très loin de ce à quoi j'assiste quand je chemine au fond de moi. ${ }^{4}$

Le dédoublement de soi en regard et existence (ou regard et désespoir) donne au second terme, objet de l'introspection, un statut à l'image du réel extérieur. La perception confère à l'objet de conscience la réalité d'un phénomène observable.

1 Charles Juliet, Joumal I, Paris, Hachette, 1978, p. 52 (note du 13 novembre 1958).

2 Ibid., p. 60 (18 février 1959).

3 Ibid., p. 93 (8 novembre 1959).

4 Ibid., p. 55 (2 janvier 1959). 
Cette perception du moi dans une distance qui le fige comme réel n'est toutefois pas continue. S'oppose à elle, tout d'abord, la force de la vie physiologique qui se continue et que le diariste ne peut nier, force qui est perçue comme celle de la positivité, de la résistance face au désespoir et à la destruction ${ }^{5}$. Ensuite et surtout, le réel extérieur s'impose à la conscience et rompt l'enchantement délétère de l'introspection:

Soudain [...] quelque chose cède en moi, et ma crispation se relâche, une sorte d'apaisement survient, je m'arrête de marcher, lève la tête, découvre le ciel, les maisons, perçois la ville autour de moi. Je vis quelques minutes d'ineffable étonnement, et un court instant, je reprends pied en moi-même. ${ }^{6}$

L'étonnement est celui du contact rétabli avec le monde, de la perception retrouvée, et de l'affirmation - temporaire - du moi, par l'intermédiaire de ce contact. Le réel se présente à nouveau sous la forme de l'observable distinct du moi, et celui-ci se confond avec sa conscience.

Deux formes de rapport à la réalité alternent ainsi : le plus souvent, la peur et la souffrance effacent la perception du monde par laquelle pourrait s'affirmer le moi, et s'imposent comme seul objet de la conscience. Par bribes, seulement, le moi a le sentiment d'exister par un contact retrouvé avec le monde extérieur.

Cette alternance, toutefois, n'est pas régulière. La perception désespérée contamine souvent l'appréhension du réel, au point de donner l'illusion d'un nouveau réel qui est, lui aussi, désespéré:

Déjà se glisse dans les heures tièdes de ces après-midi venteuses l'âcre ennui des journées d'août harassées de soleil. Déjà tout se fige. La sève d'une nouvelle mort parcourt la plaine immuable. 7

La description associe aux images de printemps celles de mûrissement et de mort, par l'intermédiaire de l'évocation d'un poison se diffusant dans la nature; les marqueurs de temps soulignent que, sous l'apparente exaltation du printemps, la mort est à l'œuvre, que sous la réalité visible une autre est perceptible. Il semble que le désespoir ait absorbé le monde extérieur. Mais

5 Ibid., p. 49 (25 octobre 1958).

6 Ibid., p. 33 (29 novembre 1957).

7 Ibid., p. 64 (3 avril 1959). Voir aussi la note du 11 août 1959, p. 80. 
86

pour le diariste la vérité est là, dans cette perception qui rapproche les étapes du cycle de la vie, qui fait apparaître sous le réel présent le réel futur, qui quête les traces du second dans le premier, et finalement reconstruit l'un en fonction de l'autre. Pour Charles Juliet, le réel est la mort. Le monde et la vie ne sont que des réalités passagères; la mort est le seul élément organisateur du réel, et finalement le seul objet de perception: en soi ou dans le monde, l'entropie est perceptible partout, tout en est la manifestation.

L'expression de la souffrance intime se développe dans ce cadre. L'exploration, en soi, de l'ennui et de l'horreur de vivre passe par le regard qui fige tout sentiment, et la perception du monde extérieur est fragmentaire ou contaminée par un sentiment de mort généralisé. Ce dernier est le principe organisateur de la perception du diariste: le canal par lequel il perçoit le réel, ou l'objet de son introspection.

\section{II}

Cette dimension statique de l'introspection va être habitée par la dynamique du temps, et plus exactement par la projection du moi dans l'avenir. Dans sa quête d'expression de soi, face à l'image omniprésente de la mort, le diariste va se construire un destin tragique.

Le désespoir connaît une progression qui peut d'abord sembler sans fin. Les notes de journal enchaînent ennui, errance en soi et désespoir toujours plus profond, tandis qu'à l'intérieur de certaines notes, l'expression du mal-être se fait par accumulation: "Tout m'humilie, me blesse, accroît mon dégoût de vivre" ${ }^{8}$. La spirale du désespoir, toutefois, n'est pas sans fond: elle est limitée par ce point final que constitue le suicide imaginé. La possibilité de mettre fin à ses jours, ou plus précisément la possibilité d'imaginer un point qui limite le désespoir et soit le négatif du présent, va structurer l'imaginaire de Charles Juliet:

Que survienne le moindre ennui, la moindre contrariété, et je pense au suicide. Comme s'il devait être ma référence constante. ${ }^{9}$

$8 \quad$ Ibid., p. 38 (25 mars 1958).

$9 \quad$ Ibid., p. 56 (29 janvier 1959). 
Le suicide offre une possibilité de fuite hors de soi et du monde, et s'impose comme une référence obligée: l'enchaînement de la contrariété et de la tentation du suicide ne permet pas de troisième terme. Ailleurs, le raisonnement se voudra plus étoffé, mais n'échappera pas à cette même relation binaire:

Cette vie plus proche de la mort que de la vie, le seul moyen de ne pas la trahir est de la refuser, de lui préférer la mort. ${ }^{10}$

Le sentiment de mort débouche sur un désir de mort, au nom d'une nécessité intime de fidélité: la vie est une trahison, une acceptation de la souffrance qui avilit; la mort en est la délivrance.

Le destin tragique du diariste se révèle dans cette opposition entre une vie misérable par essence, vouée à la souffrance, et une mort qui rende présent l'absolu:

Se suicider à vingt-quatre ans, c'est choisir la perfection, refuser de se laisser user par le temps, de voir s'éteindre sa jeunesse et tomber sa faim d'absolu. ${ }^{11}$

Deux séries d'instances s'opposent ainsi l'une à l'autre: la vie, le compromis, la souffrance et le vieillissement trouvent leur pendant dans la mort, la fidélité, la perfection, l'absolu. Les premiers sont du côté du réel; les seconds du côté du choix, du possible, de l'imaginaire, et de l'absence. Le tragique - la fiction tragique - se construit comme l'aboutissement d'une nécessité intime, en opposition au réel, et comme la seule forme possible de réalisation de soi, dans la mort. Le destin tragique est une construction imaginaire dans laquelle le désespoir trouve un accomplissement, dans laquelle le diariste s'arrache en rêve au réel quotidien, et où son existence prend sens: le suicide à venir fait du présent son passé. L'ennui de vivre qui pèse sur lui au jour le jour est éclairé par cet avenir qui fait de sa souffrance un long calvaire.

Ce chemin de croix sera aussi celui du créateur: la pensée est érosion, destruction systématique, dans l'angoisse ${ }^{12}$, et l'écriture est une lente approche de la mort, jusqu'au suicide qui n'en est que le dernier avatar. Celui qui écrit porte en lui sa propre

\footnotetext{
10 Ibid., p. 35 (15 janvier 1958).

11 Ibid., p. 73-74 (24 juin 1959).

12 Ibid., p. 42 (29 juillet 1958 ).
} 
malédiction; la révélation qu'il profère l'enchaîne toujours plus sûrement à son suicide à venir: "Je sais qu'il me faudra écrire, puis me suicider" ${ }^{13}$. L'image du poète maudit est sous-jacente: méprisé par les hommes, il ne connaît que son désespoir solipsiste, sa lucidité sans ombre ${ }^{14}$ et la condamnation à mort qui pèse sur lui. Il ne sera pas comme les autres qui "marchent dans le sens de la vie, ont donné leur consentement", mais il restera "à l'écart " ${ }^{15}$. Il connaîtra jusqu'à son dernier point la solitude mortelle qui pèse sur lui. croix :

Et, à l'extrême, le poète souffrant sera à l'image du Christ en

Le poète, c'est un peu le Christ qui, sur le calvaire, touche le fond du désespoir, mais qui, connaissant la grandeur de son sacrifice, échappe par cela même au désespoir. ${ }^{16}$

L'aventure d'exister et d'écrire glisse vers le mythe - qu'on entendra comme un récit fabuleux et exemplaire, chargé de significations sacrées. La souffrance devient, sous la plume du diariste, l'élément d'une histoire qui est celle de la découverte d'une signification - jusque-là cachée - à l'aventure de la souffrance : l'expérience du désespoir est un sacrifice, et la connaissance nouvelle que celui-ci rend possible, le sens qu'il révèle, et à la source d'un nouvel espoir. Un basculement s'opère ainsi, par le sacrifice, du désespoir à l'espoir, du non-sens à la connaissance. Le récit de soi, par la métaphore du Christ, se fait révélation: un sens peut être donné à l'aventure de souffrir, d'imaginer se tuer et de l'écrire, un sens qui est "source de vie" selon les mots du diariste ${ }^{17}$, et, par cela même, renversement total. La tentation du suicide n'est plus au coeur de la quête d'expression du désespoir, mais s'efface derrière une valeur positive, magiquement extraite du désespoir et de la souffrance. L'image du Christ et surtout le concept de sacrifice permettent cette nouvelle expression de soi qui n'est plus significative par rapport à un point futur imaginaire, mais qui tire son sens de la souffrance présente, en un renversement salvateur.

Cette métaphore mystique ne sera pas directement filée par Charles Juliet, mais le basculement qu'elle permet sera réinvesti

\footnotetext{
13 Ibid., p. 80 (17 août 1959).

14 Ibid., p. 73 (20 juin 1959).

15 Ibid., p. 117 (4 avril 1960)

16 Ibid., p. 190 (25 janvier 1962).

17 Ibid.
} 
dans une image de l'écriture ouverte sur l'existence et où s'affirme le moi ${ }^{18}$; il sera aussi reformulé, des années plus tard, dans des termes très proches de ceux cités ci-dessus, mais sans ses références sacrées:

Quand doute et angoisse et déchirement se convertissent en cette connaissance, cette joie et cette adhésion d'où naît une force grave et sereine. ${ }^{19}$

La quête de sens se clôt là, vingt ans plus tard, dans le troisième et dernier volume de journal, avec la persistance de ce renversement de l'angoisse en adhésion et affirmation de soi, par le moyen de la connaissance. C'est bien, sans ses oripeaux mystiques, la même transmutation sacrificielle qui est renouvelée. Le sens - et avec lui l'espoir, et la vie - surgit d'une construction mythique qui métamorphose la perception que le diariste a de lui-même et de son désespoir.

Pour le diariste désespéré, la souffrance et l'angoisse existentielle tenaient lieu de réel, étaient l'objet quasi unique de son introspection, figé à distance par sa conscience-regard. Elles se sont chargées de sens en s'intégrant à une histoire tragique puis mythique: celle, d'abord, du moi tenté par le suicide et dont la vie s'organisait autour de cet à-venir désespéré; celle, ensuite, du poète maudit s'enfonçant par l'écriture dans la mort; celle, enfin, du poète-Christ trouvant le sens par la connaissance de son désespoir-sacrifice, et retrouvant l'espoir avec ce sens.

Le cheminement du diariste, au long des étapes de son histoire existentielle, apparaît comme celui d'un approfondissement continuel de la dimension mythique par un emboîtement de constructions qui viennent donner une signification à l'existence et à la souffrance, jusqu'au récit de métamorphose. Mais en même temps, cette aventure existentielle et d'écriture est une redécouverte de soi et du monde, c'est-à-dire une redécouverte du réel. Celui-ci reprend son poids; la vie se révèle "drue,

18 Ibid., p. 220 (17 mars 1963); voir aussi Journal II, Paris, Hachette, 1979, p. 98 (15 février 1966).

19 Journal III, Paris, Hachette, 1982, p. 27 (février 1969). 
90

lourde", mais aussi "souple, légère "20. Le troisième volume du journal est d'ailleurs tout entier ouvert sur le monde et les autres, sur soi aussi, en une introspection sans distance où la joie que ressent le diariste est à la mesure de son "accord avec soi et avec le monde" 21 , et où son bonheur est de "tenter de restituer quelques instants de vie" 22 . Ce contact renoué avec le monde et avec soi - avec le réel - , passe, en un paradoxe apparent, par l'emboîtement de récits tragiques et mythiques, c'est-à-dire par une rupture toujours plus grande avec le réel - car seule cette rupture permet de comprendre la mort. De l'indistinction de la perception désespérée à la redécouverte du réel dans son évidence tranquille, le détour par le récit tragique puis mythique est un détour par le sens, par la connaissance - et l'acceptation de la mort. 